\title{
Luxação tarsometatársica em cão: artrodese parcial utilizando placa em " $t$ " - relato de caso
}

\author{
Tarsometatarsal luxation in a dog: parcial arthrodesis employing a \\ "t" plate - case report
}

\section{Luxación tarsometatarsiana en perros: artrodesis parcial utilizando placa en "t" - relato de caso}

\section{Leandro Romano; ${ }^{1}$ Alexandre Schmaedecke; ${ }^{2}$ Laura Bertolacini Romano; ${ }^{3}$ Cássio Ricardo Auada Ferrigno ${ }^{4}$}

Departamento de Cirurgia da Faculdade de Medicina Veterinária e Zootecnia da Universidade de São Paulo (FMVZ/USP). São Paulo, SP, Brasil

\section{Resumo}

Objetivo: Apresentar caso de artrodese parcial com utilização de placa óssea em " $t$ " e parafusos como tratamento de luxação de tarso em cão. Descrição: Cadela sem raça definida, três anos de idade, aproximadamente $14 \mathrm{~kg}$, foi atendida no Serviço de Cirurgia Ortopédica da Faculdade de Medicina Veterinária da Universidade de São Paulo (FMVZ/ USP), São Paulo, SP, com histórico de claudicação. Ao exame físico, o animal apresentava impotência funcional de membro posterior esquerdo, aumento de volume e sensibilidade dolorosa. À palpação do membro notou-se mobilidade anormal, creptação presente, dor severa e instabilidade na região da articulação tarsometatársica. O exame radiográfico revelou luxação tarsometatársica com desvio do eixo axial e perda parcial da relação articular tarsometatársica. Com base nos sinais clínicos, radiográficos e laboratoriais, marcou-se a cirurgia. A placa em " $t$ " foi posicionada de maneira que os dois parafusos proximais ficassem fixados no tarso e os quatro parafusos distais no terceiro metatarsiano. No pós-operatório imediato foi adotado penso esparadrapado com talas laterais por 60 dias. Em dois meses de pósoperatório foi dado alta ao paciente. Conclusões: A artrodese total é atualmente o procedimento cirúrgico mais utilizado em luxações do tarso de cães. Porém, a artrodese parcial pode ser considerada por apresentar pouca complicação e pequeno risco de falha do implante, com preservação da integridade de articulações não-afetadas.

Palavras-chave: Tarso animal. Articulações tarsianas. Luxações. Atrodese. Cirurgia. Cães.

'Pós-graduando do Departamento de Cirurgia da FMVZ/USP. CRMV-SP 15299

${ }^{2}$ Pós-graduando do Departamento de Cirurgia da FMVZ/USP. CRMV-PR 4439

${ }^{3}$ Médica Veterinária Autônoma. CRMV-SP 15110

${ }^{4}$ Professor Doutor do Departamento de Cirurgia da FMVZ/USP. CRMV-SP 6835 


\section{Introdução}

As lesões distais dos membros pélvicos são comuns em cães e gatos, e podem ser constituídas de fraturas, lesões ligamentares e de combinações entre elas. A extremidade distal do membro pélvico constitui-se de uma estrutura complexa e altamente crítica, e quanto maior e mais atlético for o animal, mais devastadoras serão as lesões nesta área. ${ }^{1,2}$

Artrodese é a abolição dos movimentos de uma articulação, mediante a fusão das superfícies opostas numa unidade óssea sólida. Comumente, é utilizada como procedimento de salvamento, para que seja restaurado o uso de uma articulação dolorosa ou instável, e que não respondeu ao tratamento mais conservador. A artrodese é indicada nos casos em que a movimentação contínua da articulação for mecânica ou fisiologicamente contraproducente, e não houver possibilidade do reparo menos drástico e mais anatômico. ${ }^{1,3-5}$

As indicações mais comuns para a prática da artrodese em Medicina Veterinária podem ser classificadas em três categorias de lesão: traumática, evolutiva e congênita. As lesões traumáticas consistem de fraturas e lesões ligamentares, acompanhadas ou não de deslocamento. Estes eventos envolvem estruturas em que o reparo primário conduz à instabilidade crônica, afecção articular degenerativa e dor, como em lesões do tarso com comprometimento do tecido ósseo ou ligamentar. As principais lesões evolutivas podem ser abrangidas pela denominação artrose, já as moléstias congênitas são aquelas não passíveis de reparo primário. ${ }^{1,4-6}$

O procedimento cirúrgico em si, independentemente da articulação afetada, envolve certos princípios. A estabilidade é de fundamental importância, pois as estruturas mecânicas de um membro envolvidas foram constituídas para que movimentos ocorram, portanto, a biomecânica está trabalhando contra a imobilidade no ponto de fixação. ${ }^{1,7} \mathrm{O}$ tipo de fixação e a técnica cirúrgica devem ser avaliados criteriosamente, sendo desejável o emprego de compressão e remoção da cartilagem articular para que aumente o contato ósseo e fique facilitada a união precoce. ${ }^{1,7}$
Quando um ponto de movimento é artrodesado, o aumento das pressões é transferido para os ossos e articulações adjacentes. O cirurgião deve estar ciente para, dessa maneira, antecipar e prever possíveis falhas. ${ }^{1,8,9}$

A artrodese total é o método cirúrgico mais utilizado para estabilização de articulações distais, ${ }^{1}$ porém, a artrodese parcial é considerada o tratamento de escolha para luxações e subluxações trasometatársicas ou intertarsal nos cães. ${ }^{3}$

Artrodese seletiva da articulação tarsometatársica é um procedimento cirúrgico complicado, entretanto, muitos métodos de fixação têm sido reportados. $^{5}$

Dentre inúmeras técnicas possíveis, podem-se citar o uso de parafuso tipo lag (DOVERSPIKE; VASSEUR, ${ }^{10} 1991$, GORSE et al., ${ }^{11} 1991$, DYCE,${ }^{12}$ 1996), placa óssea lateral (KLAUSE et al., ${ }^{13} 1989$, SUMMER-SMITH; KZUMA, $\left.{ }^{14} 1989\right)$, placa óssea medial, placa óssea dorsal (KLAUSE et al., ${ }^{13} 1989$ ), fixador externo linear (KLAUSE et al., ${ }^{13} 1989$, BENSON; BOURDRIEAU, ${ }^{4} 2002$ ), fixador externo circular (TROSTEL; RADASCH, ${ }^{15} 1998$, LEWIS et al., ${ }^{16}$ 1999) e uma combinação entre parafuso tipo lag e fixador externo (KLAUSE et al., ${ }^{13} 1989$ ).

Artrodese total do tarso envolve a fusão das articulações talocrural, intertarsal e tarsometatársica, sendo considerado o melhor procedimento quando a artrodese talocrural é necessária. ${ }^{5,11,13,17}$

Injúrias na articulação do tarso no cão comumente envolvem os ligamentos colaterais, plantar ou ambos. ${ }^{3}$

O principal objetivo na realização da técnica da artrodese é a identificação e eliminação da causa primária de dor e instabilidade, obtenção de fixação rígida do implante e a promoção do retorno da função do membro afetado. Para isso, a estabilização da fratura, a remoção da cartilagem articular, a revascularização tecidual e a estimulação da formação de calo ósseo precoce são eventos importantes para o sucesso do procedimento. ${ }^{18,19}$ 
A padronização da escolha de implantes por meio do método de escores descrito por Hulse ${ }^{20}$ auxilia o cirurgião a lançar mão do material adequado, a fim de prevenir falhas que possam culminar no insucesso cirúrgico.

Como alternativa no tratamento e para que haja maior garantia de sucesso, além de uma fixação rígida do implante, deve-se explorar promotores de crescimento que proporcionem osteoindução acelerada e conseqüente consolidação óssea precoce. Enxerto de osso córtico-esponjoso autógeno é uma alternativa eficaz, todavia, enxertos exógenos como BMP ou PRP são uma alternativa viável a esses casos, principalmente quando a quantidade de auto-enxerto for insuficiente. Embora amplamente utilizados para o tratamento de união retardada e não-união óssea, são indicados também nos casos de artrodese por acelerar a cicatrização e diminuir as taxas de complicações quando comparados a tratamentos convencionais. ${ }^{1,21}$

Owen $^{2}$ (2000) utilizou fixação externa para artrodese na articulação do tarso em 17 gatos. A eficiência quanto à redução da incisão e menor manipulação dos tecidos é uma vantagem a se considerar, porém, a rotação externa, migração dos pinos, quebra do implante, lise óssea e osteomielite foram descritos como complicantes em potencial. ${ }^{2}$

Harasen $^{9}$ (1999) tratou a luxação do osso central do tarso, com parafuso tipo "itálico" intraarticular, e obteve retorno da função do membro após oito semanas.

McKee et al. ${ }^{5}$ (2004) utilizaram placa óssea especialmente desenhada para a articulação tíbiotársica lateral ou medial em 13 cães. Acreditam que o acesso medial ou lateral é menos traumático que o dorsal, e as forças aplicadas sobre a placa são menores. ${ }^{5}$

Artrodese intertarsal e tarsometatarsal usando placas de compressão dinâmicas de 2.0/2.7 $\mathrm{mm}$ ou $2.7 / 3.5 \mathrm{~mm}$ foram utilizadas por Fettig et al. ${ }^{3}$ (2002) em quatro cães, conseguindo excelente redução anatômica e fácil fixação. Esses autores consideram a artrodese parcial como tratamento de escolha para luxações e subluxações da articulação intertarsal no cão. ${ }^{3}$

Estudos retrospectivos vêm sendo desenvolvidos com resultados satisfatórios em diferentes técnicas apresentadas: Muir ${ }^{22}$ (1999) realizou um estudo retrospectivo em oito cães com subluxação tarsometatársica. Fraturas secundárias à moléstia foram encontradas em seis cães, particularmente no quarto osso do tarso e no quinto metatársico proximal. As causas mais comuns da injúria articular incluíam saltos, quedas ou trauma direto. Artrodese parcial com estabilização por placa e parafusos adicionada a enxerto ósseo foi utilizada após remoção da cartilagem articular. ${ }^{22}$ Uma consolidação óssea progressiva foi notada.

Dyce $^{12}(1997)$ avaliou a artrodese parcial da articulação do tarso em dez cães. Concluiu que a recuperação era adequada após 28 semanas da cirurgia e que o uso de placa óssea é um implante para estabilização desse tipo de injúria. Wilke et al. ${ }^{23}$ (2000) realizaram artrodese intertarsal e tarsometatársica usando acesso plantar em três cães, descrevendo que o acesso plantar é relativamente fácil e que possui um potencial de fadiga do implante menor do que em outras técnicas anteriormente descritas. Allen et al. ${ }^{24}$ (1993) estudaram 36 articulações do tarso instáveis, utilizando artrodese calcaneoquartal em todos os animais e apresentando resolução completa do problema em $81 \%$ dos casos.

\section{Relato de Caso}

Relata-se caso de cão, fêmea, sem raça definida, três anos de idade, com aproximadamente $14 \mathrm{~kg}$, atendido no Serviço de Cirurgia Ortopédica da Faculdade de Medicina Veterinária da Universidade de São Paulo (FMVZ/USP), São Paulo, SP, com histórico de claudicação de apoio constante em membro posterior esquerdo. $\mathrm{O}$ proprietário relatou trauma por mordedura no membro com evolução de dois dias e sem tratamento até aquele momento.

Ao exame físico, o animal apresentava impotência funcional de membro posterior esquerdo, aumento de volume e sensibilidade dolorosa. À 
palpação do membro notou-se mobilidade anormal, creptação presente, dor severa e instabilidade na região da articulação tarsometatársica, sugestivo de luxação dessa articulação. Ferida puntiforme correspondente à lesão traumática por mordedura foi encontrada na região medial do membro, sem hemorragia ou secreção presente.

Um exame radiográfico em projeções ortogonais revelou luxação tarsometatársica com desvio do eixo axial e perda parcial da relação articular tarsometatársica correspondente aos quarto e quinto metatarsianos e irregularidade óssea subcondral (Figuras 1 e 2).

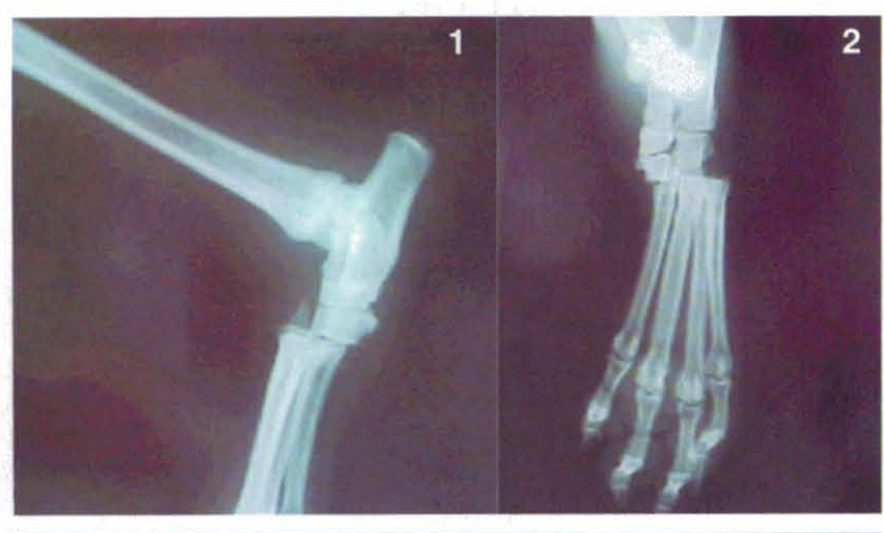

Figuras 1 e 2 - Imagens radiográficas em projeções ortogonais, demonstrando luxação tarsometatársica com deslocamento cranial

$\mathrm{O}$ animal foi medicado com meloxican na dose de $0,1 \mathrm{mg} / \mathrm{kg} \mathrm{SID}$, enrofloxacina na dose de $5 \mathrm{mg} / \mathrm{kg}$ BID e tramadol na dose de $1 \mathrm{mg} / \mathrm{kg}$ SID pela via oral. $\mathrm{O}$ tratamento paliativo com uso de penso foi instituído.

Com base nos sinais clínicos, radiográficos e laboratoriais, o diagnóstico presuntivo foi confirmado e marcou-se a cirurgia para o quinto dia após o atendimento inicial.

No procedimento anestésico o animal foi prémedicado com acepromazina $(0,05 \mathrm{mg} / \mathrm{kg})$ e meperidina $(5 \mathrm{mg} / \mathrm{kg})$ pela via intra-muscular. A indução foi feita com propofol $(5 \mathrm{mg} / \mathrm{kg})$ pela via endovenosa e a manutenção anestésica com isoflorano pela via inalatória. Foi usado anestesia epidural com tramal $(1 \mathrm{mg} / \mathrm{kg})$, associação de lidocaína $(5 \mathrm{mg} / \mathrm{kg})$ e bupivacaína $(2 \mathrm{mg} / \mathrm{kg})$.

Após o animal ter sido devidamente preparado para cirurgia asséptica, as articulações foram expostas através de incisão da pele em abordagem dorsal, os tendões flexor digital e extensor foram alternadamente afastados lateral e medialmente para que fosse possível o debridamento da cartilagem articular. A placa em " $t$ " foi posicionada de maneira que os dois parafusos proximais ficassem fixados no tarso e os quatro parafusos distais no terceiro metatarsiano (Figura 3).

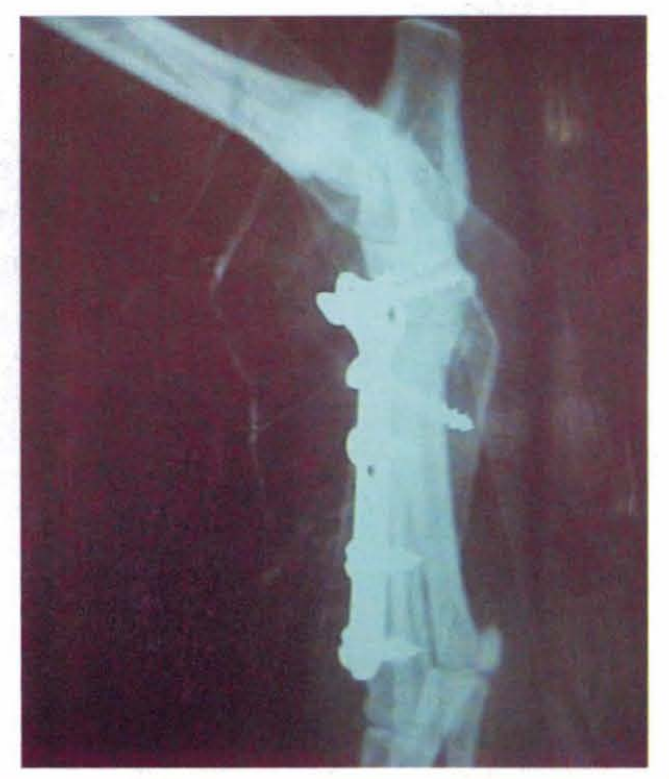

Figura 3 - Imagem radiográfica em projeção médio-lateral apresentando artrodese parcial do tarso com placa em " $\mathrm{t}$ "

Utilizou-se enxerto cortical de asa de ílio em quantidade suficiente para promover uma aceleração na consolidação óssea. Após sutura de musculatura em ponto simples separado e subcutâneo com vycril 2,0 , realizou-se a sutura da pele com nylon 2,0 em ponto simples separado.

No pós-operatório imediato foi adotado o uso de penso esparadrapado com talas laterais, mantido por 60 dias. Foi prescrito cefalexina $(30 \mathrm{mg} / \mathrm{kg}) \mathrm{BID}$, meloxican $(0,1 \mathrm{mg} / \mathrm{kg})$ SID e tramadol $(1 \mathrm{mg} / \mathrm{kg}) \mathrm{SID}$ pela via oral. 
Com evolução de 15 dias, os pontos foram retirados e notou-se leve instabilidade local, no entanto, com apoio adequado do membro. Após 30 dias, a melhora na marcha era evidente e não havia dor à palpação da região operada. Com 60 dias de pósoperatório, a bandagem foi retirada, as medicações suspensas e a alta do paciente com evolução e recuperação cirúrgica foram consideradas adequadas.

\section{Conclusões}

Em relação às luxações e subluxações dos ossos do tarso no cão, inúmeros tratamentos são propostos. Claudicação em decorrência de injúrias ligamentares, sobrecarga mecânica e traumatismo agudo são provavelmente as causas mais comuns nesse tipo de moléstia.

O uso de materiais anatomicamente desenhados para essas articulações tornam o procedimento menos drástico, promovendo o retorno precoce da função do membro afetado.
Independente do implante escolhido, do uso de promotores de crescimento e de técnicas cirúrgicas corretamente realizadas, a meta primária nestes casos é a rígida fixação do implante e a completa reparação óssea para que seja restaurado o uso do membro com uma articulação, anteriormente dolorosa e instável.

A artrodese total é, hoje em dia, o procedimento cirúrgico mais utilizado, contudo, a artrodese parcial pode ser considerada de fácil aplicação, com baixo índice de complicações e pequeno risco de falha no implante, sendo um tratamento recomendável neste tipo de moléstia.

\section{Agradecimentos}

Aos pós-graduandos do Departamento de Cirurgia da FMVZ/USP Daniel Castelo Branco Bacarin, Leonardo Pereira Lima e Silmara Bonomi da Silva pelos comentários e sugestões durante o desenvolvimento do presente relato.

\section{Abstract}

Objective: To report on a case of partial arthrodesis employing a " $t$ " bone plate and screws, for treatment of tarsal luxation in a dog. Description: Female dog, mixed breed, three years old, approximately $14 \mathrm{~kg}$, examined at the Serviço de Cirurgia Ortopédica da Faculdade de Medicina Veterinária da Universidade de São Paulo (FMVZ/USP), (Surgical Orthopedic Service of the School of Veterinary Medicine and Animal Science of the University of São Paulo), São Paulo, SP, Brazil, with a history of lameness. Upon physical examination, the left hindlimb was nonweightbearing, enlarged and painful. Palpation revealed crepitus, severe pain and instability of the tarso-metatarsal joint. Radiology revealed tarso-metatarsal luxation with axial deviation and partial loss of the tarso-metatarsal articular relationship. Based on the clinical, radiological and laboratorial findings, surgery was scheduled. The " $t$ " plate was placed in such a manner that both proximal screws were fixated to the tarsus and the four distal screws were fixated to the third metatarsus. Lateral splints were employed in the early post-operative period and kept for 60 days. The patient was released from treatment after two months. Conclusions: Today, total arthrodesis is the most common surgical procedure for treatment of tarsal luxation in dogs. However partial arthrodesis can be considered due to its few complications and low risk of implant failure, with preservation of the integrity of non affected joints.

Keywords: Tarsus, animal. Tarsal joints. Dislocations. Arthrodesis. Surgery. Dogs. 


\section{Resumen}

Objetivo: Presentar un caso de artrodesis parcial con utilización de placa ósea en " $t$ " y tornillos como tratamiento para la luxación del tarso en perros. Descripción: Una perra sin raza definida, de tres años de edad y aproximadamente 14 kilos, fue atendida en el Servicio de Cirugía Ortopédica de la Facultad de Medicina Veterinaria de la Universidad de São Paulo (FMVZ/USP), São Paulo, SP,Brasil, con un histórico de claudicación. En el examen fisico el animal presentaba impotencia funcional del miembro posterior izquierdo, aumento de volumen y sensibilidad dolorosa. En la palpación del miembro se notó una movilidad anormal, crepitación presente, dolor severo e inestabilidad de la región de la articulación tarsometatarsiana. El examen radiográfico reveló luxación tarsometatarsiana con desvio del eje axial y pérdida parcial de la relación articular tarsometatarsiana. Fundamentado en señales clínicos, radiográficos y de laboratorio, se marcó la cirugía. La placa en " $t$ " se posicionó de tal manera que los dos tornillos proximales permaneciesen fijados en el tarso y los cuatro tornillos distales en el tercer metatarsiano. En el pos operatorio inmediato se adoptó un vendaje de esparadrapo con tablillas laterales durante 60 dias. En dos meses de pos operatorio se le dio alta al paciente. Conclusiones: La artrodesis total es actualmente el procedimiento quirúrgico más utilizado en luxaciones del tarso en perros. Sin embargo la artrodesis parcial puede ser considerada al presentar poca complicación y pequeño riesgo de fallos en el implante, con preservación de la integridad de articulaciones no afectadas.

Palabras-clave: Tarso animal. Articulaciones tarsianas. Luxaciones. Artrodesis. Cirugia. Perros

\section{Referências}

1. LESSER, A. L. Artrodese. In: SLATTER, D. Manual de cirurgia de pequenos animais. São Paulo: Manole, 1998. p. 2228-2242.

2. OWEN, M. A. Use of contoured bar transhock external fixators in 17 cats. Journal of Small Animal Practice, v. 41, p. 440-446, 2000.

3. FETTIG, A. A. et al. Intertarsal and tarsometatarsal arthrodesis using $2.0 / 2.7 \mathrm{~mm}$ or $2.7 / 3.5 \mathrm{~mm}$ hybrid dynamic compression plates. Journal of the American Animal Hospital Association, v. 38, p. 364-369, 2002.

4. BENSON, J. A., BODRIEAU, R. J. Severe carpal and tarsal shearing injuries treated with an immediate arthrodesis in seven dogs. Journal of the American Animal Hospital Association, v. 38, p. 370-380, 2002.

5. McKEE, W. M. et al. Pantarsal arthrodesis with a customized medial or lateral bone plate in 13 dogs. Veterinary Record, v. 154, p. 165-170, 2004.

6. GARRETT Jr., W.E.; BEST, T.M. Anatomy, physiology and mechanics of skeletal muscle. In: SIMON, S. R. (Ed.). Orthopaedic basic science. 2. ed. Rosemond: American Academy of Orthopaedic Surgeons, 2000. p. 683-716.
7. PIERMATTEI, D. L., FLO, G. L. Handbook of small animal orthopedics and fracture repair. 3. ed. Philadelphia: Saunders, 1997. p. 636-641.

8. DEE, J. F. Fraturas em galgos de corrida. In: BOJRAB, M. J. Mecanismos da moléstia na cirurgia de pequenos animais. São Paulo: Manole, 1996. p. 1220-1236.

9. HARASEN, G. L. Fracture-luxation of the central tarsal bone. Canadian Veterinary Journal, v. 40, p. $195,1999$.

10. DOVERSPIKE, M; VASSEUR, P. B. Clinical findings and complications after talocrural arthrodesis in dogs: experience of six cases. Journal of the American Animal Hospital Association, v. 27, p. 553-559, 1991.

11. GORSE, M. J. et al. Tarsocrural arthrodesis: longterm functional results. Journal of the American Animal Hospital Association, v. 27, p. 231-235, 1991.

12. DYCE, J. Athrodesis in the dog. In Practice, v. 18, p. 267-279, 1996.

13. KLAUSE, S. E. et al. Tarso-crural arthrodesis: complications and recommendations. Veterinary and Comparative Orthopedics and Traumatology, v. 3, p. 119-124, 1989. 
14. SUMNER-SMITH, G.; KZUMA, A. A technique for arthrodesis of the canine tarsocrural joint. Journal of Small Animal Practice, v. 30, p. 65-67, 1989.

15. TROSTEL, C. T.; RADASCH, R. M. Tarsocrural arthrodesis: a clinical report using a circular external fixator. Veterinary and Comparative Orthopedics and Traumatology, v. 11, p. 193-196, 1998.

16. LEWIS, D. D. et al. Initial clinical experience with the IMEX circular external skeletal fixation system. Veterinary and Comparative Orthopedics and Traumatology, v. 12, p. 108-117, 1999.

17. DECAMP, C. E. et al. Pantarsal arthrodesis in dog and cat: 11 cases (1983-1991). Journal of the American Veterinary Medical Association, v. 203, p. 1705-1707, 1993.

18. ROBEllo, G. T., ARON, D. N. Delayed and nonunion fractures. Seminars in Veterinary Medicine and Surgery: Small Animal, v. 7, p. 98104, 1992.
19. FINKEMEIER, C. G. Bone graft and bone graft substitutes. Journal of Bone and Joint Surgery, v. 84 , p. $454-464,2002$.

20. HULSE, D. A. et al. Decision making in fracture management. In: FOSSUN, T. W. Small animal surgery. Saint Louis: Mosby, 1997. p. 730-732.

21. SCHMAEDECKE, A. et al. Tratamento cirúrgico de fraturas em cães. Revista de Educação Continuada do CRMV-SP, v. 6, p. 74-82, 2003.

22. MUIR, P. Tarsometatarsal subluxation in dogs: partial artrodesis by plate fixation. Journal of the American Animal Hospital Association, v. 35, p. 155-162, 1999.

23. WILKE, V. L. et al. Intertarsal and tarsometatarsal arthrodesis using a plantar approach. Veterinary and Comparative Orthopedics and Traumatology, v. 13, p. $28-33,2000$.

24. ALLEN, M. J. et al. Calcaneoquartal arthrodesis in the dog. Journal of Small Animal Practice, v. 34, p. 205-210, 1993. 